\title{
Chapter 2 \\ The Need for a Common Ground for the IoT: The History and Reasoning Behind the IoT-A Project
}

\author{
Alessandro Bassi and Sebastian Lange
}

The Internet of Things concept has evolved rapidly in recent years. It can be seen as an umbrella term for interconnected technologies, devices, objects and services. Nevertheless, after many years of heavy discussion, there is still no clear and common definition of the concept. And yet the application scenarios and market opportunities offered by objects communicating actively and autonomously extend far beyond the foreseeable horizon.

Looking at websites such as kickstarters.com and indiegogo.com, new applications and services envisaged by innovators and researchers are astonishing and clearly show the vast opportunities our future society will be confronted with.

The concept of IoT as introduced in Chap. 1 emerged primarily from the convergence of different technological developments and fields. In particular, it builds on the emergence of innovative enabling functionalities that stem from identification technologies such as RFID and bar codes, as well as from the development of networked sensors and actuators. In the early 2000s, RFID technology was developed and rolled out mainly across the logistics sector for tracking and tracing goods. At the same time, research was conducted on sensor networks and miniaturized smart systems. Sensors were becoming increasingly small and computing power dramatically increased. Nevertheless, innovative solutions were always developed for specific application cases, and there was no true interoperability and interconnectivity between different application areas.

For instance, in some fields such as manufacturing and logistics, communication and tagging solutions are well-established as they provide a clear business benefit in terms of asset tracking and supply chain management. However, the same solutions

\author{
A. Bassi $(\bowtie)$ \\ Alessandro Bassi Consulting, 3, Avenue de Cannes, 09160 Juan Les Pins, France \\ e-mail: alessandro@bassiconsulting.eu \\ S. Lange \\ Deloitte \& Touche GmbH, Kurfürstendamm 23, 10719 Berlin, Germany \\ e-mail: sebastian.lange@web.de
}


do not apply for other fields such as domotics, where business synergies could provide services with clear added-value benefits.

As the IoT domain covers such a wide spectrum of application fields with very little in common, the development cycles and technologies used can be completely different. Often, early technological developments are driven by visionary small and medium-sized enterprises (SME) that are able to innovate faster and to catch emerging trends. However, the target is usually a product or service with a narrow scope, and as the focus and window of opportunity are slim, the solutions developed are usually non-interoperable, and while successful, they do not produce a common abstract infrastructure capable of marking significant progress in the whole field. The same holds true for large industry companies that often develop specialized solutions for dedicated business opportunities without implementing generally applicable concepts.

Therefore, current solutions can still be seen as island solutions, implementing some sort of "INTRAnet of Things" rather than an "INTERnet of Things".

While quite logical at this point, in the long term, this situation is unsustainable. Today, we can observe a similar situation to that in the networking field, where several solutions emerged at its infancy but were subsequently abandoned in favour of a unified communication infrastructure, the TCP/IP protocol suite.

The emergence of a common "lingua franca" for the IoT domain, representing the narrow central point in the Internet protocol suite, is a prerequisite for quick and pervasive development of innovative solutions that can leverage different technologies developed for different targets in different application domains.

After much discussion about the core concepts of the IoT for several years, in 2009 a group of researchers from more than 20 large industrial companies and research institutions joined forces to lay the foundation for the much needed common ground or a common "architecture" for the Internet of Things: the IoTArchitecture project (IoT-A) was born. IoT-A has become the European Commission's flagship project in the European Union's Seventh Framework Program for Research and Development with respect to establishing an architecture for the Internet of Things.

Leaving aside business considerations, and considering only the technical point of view, it was clear for the project partners that the existing solutions did not address the scalability requirements of a future IoT, both in terms of communication between smart devices and the orchestration and management of complex services. Furthermore, the IoT domain comprises several different governance models, which are often incompatible. This leads to a situation where privacy and security are treated on a per case and per legislation basis, retrofitting solutions to existing designs - this severely hampers portability, interoperability and deployment.

Of course, the spread of the IoT domain is so huge that it would be naive to consider a "one-size-fits-all" protocol, such as IP, or even a single layer where interoperability between all sorts of smart device communication can take place. However, it soon became clear that within this area, there was a need for a common ground in a more abstract layer. 
We are convinced that different classes of devices will always co-exist. Taxonomies can be created according to different principles, such as critical or non-critical, or distributed or centralised. These classes can foster different profiles according to the specific needs and requirements of applications and domains.

As it is impossible to specify one single design pattern that can satisfy all application domains, the common ground has to be found at a more abstract level. We believe that the identification of a reference model for the entire IoT domain will provide the common ground. By reference model we mean an abstract framework that comprises a minimal set of unifying concepts, axioms and relationships for understanding significant relationships between the entities of an environment. This framework should enable the development of specific architectures which may have different levels of abstraction. At this level of abstraction we are independent of specific standards, technologies, implementations, or other concrete details.

This high-level work then drives the realisation of a framework for identifying specific reference architectures that subsequently describe both essential building blocks as well as design choices for dealing with conflicting requirements regarding functionality, performance, deployment and security. Interfaces need to be standardised, and best practices need to be provided in terms of functionality and information usage.

The central decision of the IoT-A project was to base its work on the current state of the art, rather than applying a clean slate approach. As a result, common traits have been derived to form the baseline of the IoT Architectural Reference Model (ARM). This has the major advantage of ensuring that the model is backwardcompatible, as well as the adoption of established, working solutions for various aspects of the IoT.

It is no longer possible to build architectures in the lab or without real world input. IoT-A acknowledged this new reality, where the lines between R\&D, innovation and emergent technologies are blurred, at a very early stage. With the help of end users, organised into the IoT-A stakeholders group, new requirements for IoT have been collected and introduced in the main model-building process. This stakeholder group was one of the most important sources for obtaining external input as well as feedback on the current status of project work. Thus far, the stakeholder contributions have been a main feature of the project, as the stakeholder requirements collected in an initial workshop formed the basis for the initial draft of the ARM, particularly the domain model and the functional decomposition. Each building block of the ARM was then developed to meet all requirements and enable the IoT-A holistic approach. Further stakeholder workshops and questionnaires were employed to review the progress of the ARM development and to fine tune the concepts and models.

Currently, the prevailing practice domain for stakeholder engagement is largely characterized by complex and dynamic environments that cover a wide range of stakeholders, from hostile to conciliatory, from obstructive to collaborative.

This is an apt characterisation of the Internet of Things: complex and dynamic environments containing a wide range of stakeholders. As such, it is an open and 
ongoing ecology of environments, characterized by change and real-time combinatorial innovation.

For all their different backgrounds - automotive, health, logistics, retail... - the stakeholders were surprised to see that their requirements were often very similar. In their real world cases, the same principles and same abstract level required that "in this IoT world things become active participants"; the goal is a seamless chain of real-time tracking and tracing, in which the elite of expensive high-level item tracking and the multitude of low-level items should be balanced for cost efficiency. Interoperability was validated by the stakeholders in the independently generated use cases as the number one requirement.

The next chapter introduces the ARM in detail, its language and terminology, as well as its beneficial role in the IoT application development process.

Open Access This chapter is distributed under the terms of the Creative Commons Attribution Noncommercial License, which permits any noncommercial use, distribution, and reproduction in any medium, provided the original author(s) and source are credited. 